their statement, that while the colour produced by boiling liquor potassae with diabetic urine is a "rich claret" that which ensues with grape-sugar, potash, and distilled water is a "dark sherry colour." To this statement I shall have again to refer.

It will be observed, also, that Messrs. Moore and Palmer state, that when healthy urine is boiled with liquor potassæe in excess, no change of colour ensues; that Dr. Golding Bird asserts that boiling with liquor potassæe rather tends to bleach non-saccharine urine than to deepen its colour, and that Dr. Bence Jones affirms that some deepening of colour may ensue when no sugar is in the urine.

We have here, then, a remarkable difference of opinion.

We will now proceed to ascertain how far the opinions and statements above quoted are confirmed by some additional observations and experiments.

1st Experiment.-Liquor potassæ was applied in the ordinary manner to nearly colourless solutions of thirty-six different samples of cane-sugar, obtained either direct from the hogshead or from grocers. In each case the solutions were deepened several degrees in colour, and in general they assumed the tint of brown sherry.

2nd Experiment.-Liquor potassæe was added to a colourless solution of cune-sugar, obtained trom the sugar-cane itself. In this case, also, the solution acquired the colour of sherry.

3rd Experiment.-Twelve different solutions of white or refined cane-sugar were tested with liquor potassæ in the usual manner: five of the solutions were scarcely affected at all, but in the remainder a slight coloration became perceptible.

4 th Experiment.-Solutions of twelve different syrups, made of white sugar similarly treated, all became slightly browned, and in general to a greater extent than in the previous experiment.

5th Experiment. - Liquor potassæe was added to three different and colourless solutions of grape-sugar in distilled water, containing respectively one grain, half a grain, and a quarter of a grain of sugar to the ounce: the first, on the application of heat, became of the colour of pale sherry; the second, of a straw colour, and the third was scarcely perceptibly coloured.

$6 t h$ Experiment.-Three solutions of caustic potash, urine, and sugars, of the same strength as the previous solutions, were prepared. When boiled, the first presented the colour of dark-brown sherry, the second of a pale sherry, and the third was straw-coloured.

The colour of a portion of the same urine boiled with potash alone was between that of the two last solutions.

7 th Experiment.-Twelve non-saccharine urines were subjected to the combined action of liquor potassæ and heat; in each case the urine became very perceptibly darker than before.

In order that the change in the colour of the tested urine may be the more clearly seen, it should be contrasted with an equal quantity of the same urine which has not been sub. mitted to the test, and contained in a tube of the same size as that employed in the experiment with the solution of urine and potash.

8th Experiment.--Several solutions of diabetic urine and potash were submitted to the action of heat; the change in the appearance of the urine in each case was very marked, a dark reddish-brown colour being developed.

From the above experiments, several conclusions may be deduced. It is generally stated by chemists that cane may be readily distinguished from grape sugar by means of liquor potassæ. By experiments $1 \& 2$ it is shown that this is not the case, so far as sugar, as procured direct from the cane itself, and as well as the ordinary brown sugars of commerce are concerned-but that the reaction with the alkali is to a very great extent the same in the two cases.

This identity of result is, no doubt, occasioned by the circumstance, that in the cane the two varieties of sugar are generally associated together.

By the third experiment it appears, however, that liquor potassæe has but little action on well refined cane-sugar, and therefore by its means this form of sugar may be discriminated from grape-sugar.

By the fourth experiment, again, it is shown that even the moderate degree of heat employed in reducing lump-sugar to the condition of syrup has a tendency to convert cane into grape sugar, the conversion being more complete as the temperature is augmented.

By the fifth experiment it is seen that not less than a quarter of a grain of diabetic sugar to the ounce of distilled water can be satisfactorily detected by the liquor-potassæ test.

And by the sixth experiment it appears that not less than half a grain of grape-sugar to the ounce of urine can with certainty be recognised by means of that test.

Now, if the quantity of urine passed in the twenty-four hours in health be considered to average about fifty ounces, and as where the urine is saccharine this quantity is considerably increased, it follows that as much as half a drachm of diabetic sugar may in some cases be eliminated by the kidneys in a day-an amount sufficient to indicate an impairment of the functions of assimilation, so serious as to affect considerably the powers of life, and yet elude detection by means of the liquor-potassae test.

By the seventh experiment, it is shown that the action of liquor potassee on non-saccharine urine is to darken it very perceptibly, and that therefore it is incorrect to say, with Messrs. Moore and Palmer, that this test does not effect any change of colour in healthy urine; or with Dr. Golding Bird, that "boiling with liquor potassæ rather tends to bleach non. saccharine urine than to deepen its colour."

The observation of Dr. Bence Jones, that " some deepening of colour may ensue when no sugar is in the urine," was a step in the right direction, but it failed to go far enough.

A moderate increase in the colour of the urine, when boiled with liquor potass, is no proof, therefore, of the presence of grape-sugar in urine.

Finally, by the eighth experiment, it is shown that the colour of saccharine urine is very remarkably affected by boiling with liquor potassæ. Now, as healthy urine is deepened in colour, to a certain extent, by the same means, and as the depth of colour in the case of diabetic urine is so much greater than that of a solution of grape-sugar in distilled water similarly treated, it follows that the whole of the colour developed by the action of potash on saccharine urine, is not due entirely to the sugar present, but in part depends upon the action of the potash on some of the normal constituents of the urine itself.

It is evident therefore that we cannot safely rely upon the liquor potassæ test, unless in those cases in which the indications afforded by it are of a very marked character, and also that by its means we are unable to detect in the urine, with certainty, the presence of an amount of sugar which, continuing " de die in diem," is sufficient to indicate serious derangement of the assimilative functions, and to effect injuriously the health.

Park-street, Grosvenor-square, Feb. 1, 1851 .

(To be continued.)

\section{CASE OF NEARLY IMPERFORATE HYMEN IN A PRIMIPARA.}

By JAMES SEATON, Assistant to Edwin Foster, M.D.

ON the evening of Thursday, the 23rd Jan., I was called to attend a woman, aged twenty-five, in labour of her first child. On examination, it was found impossible to introduce the finger, in consequence of the entrance to the vagina being obstructed by the hymen in a nearly imperforate state; the membrane felt very firm and unyielding, convincing me that it would offer considerable resistance to the birth of the foetus. The only communication with the vagina was by means of an orifice situated in the anterior front of the membrane, and not large enough to admit the point of the finger.

In these circumstances, Dr. Foster having examined the patient,-although believing, from the unyielding character of the obstruction, that some operative procedure would ultimately be necessary,-judged that the better plan would be to trust to the natural efforts, until it should become evident that interference was absolutely required, especially as by delay our position with regard to operating would be in no way injured, but rather improved, as, after the hymen became distended, it would be rendered more easy of division. As the patient was suffering from ineffectual pains which had continued for several days, it was judged proper to administer a dose of opium, after which we left, her.

The patient remained pretty free from pain until the morning of Sunday, the $26 \mathrm{th}$, when I found her suffering strong labour-pains, at intervals of a few minutes, and on examination found the hymen partially distended, from the pressure of the liquor amnii. I remained with the patient about two hours, during which the hymen gradually became more fully distended, and at the end of that period Dr. Foster took charge of the case. About two hours after he arrived, the membrane being then very much stretched, he contrived, with 
some difficulty, to insinuate his finger fairly through the orifice into the vagina, when, partly from the pressure exerted on the membrane by the contents of the uterus, and partly from its being partially torn in the introduction of the finger, it was completely ruptured with surprising facility. In about fifteen minutes afterwards the child was safely delivered, and the mother has since recovered without a bad symptom.

It may be mentioned, that, on referring to Dr. Foster's case-book, I find that about five years ago the same patient was under treatment for most distressing abdominal pains, which recurred monthly, the cause of which symptoms was found to be an imperforate hymen, obstructing the flow of the menses, as, on its being divided, a complete remission of her symptoms took place.

Leeds, February, 1851.

\section{fthroical Éorírtíss.}

\section{ROYAL MEDICAL AND CHIRURGICAL SOCIETY.} Tuesday, Frb. 25, 1851.-Dr. Addison, President.

AfTER the "scene" reported in the last LANCE', the following papers were read:-

Case of Small-Pox Recurring a Third Time after Vaccination, when it Proved Fatal. By John Webster, M.D., F.R.S., \&c.

After alluding to the fact, that hooping-cough, measles, and scarlatina generally occur only once during the lifetime of an individual, exceptions, nevertheless, to the above rule, as well in these complaints as in small-pox, have been recorded by authors. Three well-marked examples of the recurrence of small-pox met with in the same family are related, one of which terminated fatally. The case especially referred to by Dr. Webster was that of H. N. N-, who had been vaccinated satisfactorily in 1827, when three months old. Notwithstanding this circumstance, he became attacked by small-pox in 1833, along with an elder brother, who had been likewise vaccinated. Both patients recovered, and nothing more was thought of the matter till 1838 , when the two lads were again attacked by variola, along with another-that is, a thirdbrother, likewise regularly vaccinated. However, all three got quite well in due time. Subsequently, Mr. H. N. N-, whose case is now just mentioned, went to India in the Company's service, where he was seized, in April last, with the usual and well-marked symptoms of small-pox, which soon became confluent, and proved fatal at Dharwarinth, on the 13th of that month; this making the third time this gentleman had been attacked by variola, although previously vaccinated.

Case of Coneluennt Small-Pox after a Third Vaccination. By Dr. A. P. Stewart.

A gentleman, twenty-five years of age, who had been five years apprenticed to a gentleman in extensive general practice in Devonshire, had been most successfully vaccinated when six months' old. Requiring to attend variolous cases, he had the operation repeated in July, 1849, without effect, and again in May, 1850, with much irritation, inflammation, and swelling of the arm, the lymph from the vesicle not communicating the disease. He came to London to begin his medical studies on the 13th of October last, having been exposed to infection the same morning. The train of premonitory symptoms set in on the 15 th, and was followed (on the $17 \mathrm{th})$ by the appearance of a closely packed eruption of papulæ all over the body. For four successive nights, mild delirium was present; but the sore throat and conjunctivitis occasioned by the pressure of vesicles were quickly relieved by the application of a solution of nitrate of silver; and the pulse, previously as high as 120 , never rose above 100 after the seventh day. The vesicles, though very numerous on the hands and feet, caused little or no pain; on the eleventh day of disease, and seventh of eruption, they were at their height and in the course of the next two days, had nearly all dried up and scaled off, with very trifling discharge. Thereafte convalescence went on rapidly. In the district where the subject of this attack resided, the popular prejudice against vaccination has always been so strong, that for a number of years it had been much neglected; and the parents are still in the habit of inoculating their children. During the last nine months, it has been practised to a very large extent, with so much secrecy as to defy the utmost efforts of the authorities to obtain legal proof, and with the effect of keeping up the disease during many months, and spreading it abroad over the whole district. It was introduced in April by a sweep boy, who took it in a town not far off, and, returning to his family, communicated it to his unvaccinated brothers and sisters. The latter, again, going to a lace-school, infected several of the other children, after which it spread rapidly in all directions. Several instances were adduced, as examples of a large number in which inoculation had entirely failed to protect those who had been subjected to it from secondary attacks; besides the case of a lady of title, whose mansion was in the infected district, and who had lately had a second attack of natural small-pox. The author then stated, as the result of his and Dr. A. Anderson's experience in the Glasgow Fever Hospital, from November, 1836, to November, 1838, that of the 126 cases admitted, 31 were vaccinated, 52 doubtful, (consisting of unvaccinated, with a sprinkling of imperfectly vaccinated patients, and 43 unvaccinated. The mortality among the "doubtful" was 1 in 3.06 , or 32.7 per cent.; among the unvaccinated, 1 in $2 \cdot 86$, or $34 \cdot 8$ per cent.; while among the vaccinated it was only 1 in 31 , or $3 \cdot 2$ per cent. The author concluded his remarks by adducing his experience in the north-west district of St. Pancras parish, during the epidemic of 1845 . Those who had been successfully vaccinated, both children and parents, though exposed night and day, in their unventilated dwellings, to the concentrated infec* tion of the disease in its worst forms, were proof against it; while those in whom the evidence of success was doubtful were not proof against the milder forms, and very often took the disease from convalescents whom they met in the open air. The author concluded, with Chomel, that "we cannot fairly exact more from vaccination than from the small-pox itself."

Dr. MaYo, in reference to the opinion of Chomel, that we had no right to expect more from vaccination than from inoculation itself, referred to an active epidemic, small-pox, which raged in Edinburgh in 1817, and which was described by Dr. Thomson. In this epidemic it was calculated that more persons had been affected by small-pox after inoculation than after vaccination, and of the first more had died, so that the difference in the protective power was in favour of vaccination over inoculation. He inquired if Dr. Gregory had seen the paper referred to. One important point in respect to vaccination was the influence of re-vaccination. What did it effect? Dr. Mayo then referred to some cases of small-pox which at first simulated measles, which occurred in the Middlesex Hospital, to show the necessity of distinguishing between what might be only an apparent and not a real repetition of a disease.

Dr. GrEGORY said that the cases detailed were interesting, and contained much which was worthy of the consideration of the Society. With respect to the first case, in which a person contracted small-pox in advanced life after vaccination, and died in India, he might remark, that the disease was more fatal in warm climates, and the case in this respect was not remarkable. The similarity of the cases, however, consisted in the statement which had been made, that these patients had small-pox three years after, and again five years after vaccination. Now, he would beg to ask Dr. Webster, who attended these cases, was he acquainted with the medical man who had the patients under his charge? He asked this, in order that it might be determined if the disease under which these patients laboured were variola or not. Certain it was that he (Dr. Gregory) never saw such cases; they never presented themselves at the Small-Pox Hospital. He must therefore be excused from doubting whether these cases were really cases of variola until the doubt had been cleared up by further information from Dr. Webster. With respect to the second case, which was one of confluent smallpox after vaccination, it really contained nothing remarkable, except indeed the fact of re-vaccination having been practised and followed by local inflammation. This showed convineingly the little value of re-vaccination. He had held this opinion for a long time past. But by far the most important part of the paper was its closing paragraph, the doctrine of Chomel, " that we had no right to expect more from vaccination than from inoculation itself." These few words involved considerations of the highest importance, and it was certain that the attention of the profession and the public must be called to the entire question, and to determine the value of the dictum in question. What, for instance, did we expect from inoculation? By it we gave the small-pox to the person inoculated. We submitted the system to a subtle and violent miasma; the system fortunately took it mildly, passed through the disease, and thus the matter ended. There might be exceptional cases to this termination, and some might have the ill luck to have small-pox a second time; but he believed few 